\title{
Performance Modeling and Analysis of AF Relaying with Hidden Nodes
}

DOI:

10.1109/WCNC.2017.7925929

\section{Document Version}

Accepted author manuscript

Link to publication record in Manchester Research Explorer

\section{Citation for published version (APA):}

Alfitouri, A., \& Hamdi, K. (2017). Performance Modeling and Analysis of AF Relaying with Hidden Nodes. In IEEE Wireless Communications and Networking Conference IEEE. https://doi.org/10.1109/WCNC.2017.7925929

\section{Published in:}

IEEE Wireless Communications and Networking Conference

\section{Citing this paper}

Please note that where the full-text provided on Manchester Research Explorer is the Author Accepted Manuscript or Proof version this may differ from the final Published version. If citing, it is advised that you check and use the publisher's definitive version.

\section{General rights}

Copyright and moral rights for the publications made accessible in the Research Explorer are retained by the authors and/or other copyright owners and it is a condition of accessing publications that users recognise and abide by the legal requirements associated with these rights.

\section{Takedown policy}

If you believe that this document breaches copyright please refer to the University of Manchester's Takedown Procedures [http://man.ac.uk/04Y6Bo] or contact uml.scholarlycommunications@manchester.ac.uk providing relevant details, so we can investigate your claim.

\section{OPEN ACCESS}




\title{
Performance Modeling and Analysis of AF Relaying with Hidden Nodes
}

\author{
Abdurrahman Alfitouri, and Khairi Ashour Hamdi \\ School of Electrical and Electronic Engineering, \\ University of Manchester, Manchester, M13 9PL, UK \\ Emails: \{Abdurrahman.alfitouri, k.hamdi\}@manchester.ac.uk
}

\begin{abstract}
In this paper, we investigate and analyse the performance of amplify and forward (AF) relay system connecting a group of independent sources to their destinations employing a random carrier sense multiple access (CSMA) protocol. The sources are isolated from their destination and the only means for them to communicate with their destination is through the common relay. Sources are arbitrarily distributed around the relay and experience Rayleigh fading channels. This paper develops a new and more accurate model for multiple access interference at both the relay and destination which takes into account random sources location and the effects of fading and thermal noise at both the relay and destination. Moreover, the effect of the hidden nodes on the system performance is considered as a randomly occurring factor. This leads to the derivation of new analytical expressions for the overall spectral efficiency (SE) which can be used to estimate the throughput of the CSMA-based relay in Rayleigh fading channels and to study the impact of the different system parameters on their efficiency. The accuracy of the new mathematical results is confirmed by Monte Carlo simulation.

Index Terms-Amplify and forward, carrier sense multiple access, moment generation function, Rayleigh fading, hidden nodes.
\end{abstract}

\section{INTRODUCTION}

Cooperative wireless networks can provide higher throughput and are considered an effective solution to coverage extension. One possible cooperative wireless network scenario is the multiple access relay, where a common relay helps multiple source nodes to communicate with their destination with the aim of improving system performance [1], [2]. Multiple access techniques are used to allow a large number of users to share the allocated frequency band in the most efficient way.

The mechanism of packet radio access schemes can be summarized as dividing the transmitted data into packets, where each packet is considered as a different user and transmitted individually. ALOHA is generally considered as the first wireless packet radio system [3]. When the transmitter is ready to send an information packet, the packet is sent to the receiver as soon as a channel between them is available. However, if several transmitters are simultaneously attempting to send their packets, interference can occur which leads to a decrease in the efficiency of system performance. Pure ALOHA, or unslotted ALOHA, is a part of the ALOHA system, where the starting time of transmission for each transmitter, is randomly chosen [4]. However, if the transmitter checks whether the channel is available or busy with other transmissions, before it sends its signal, this can avoid interference. If the channel is sensed idle, the user will send its signal, otherwise it will wait. This is called carrier sense multiple access (CSMA), which lead to a more efficient system compared to the ALOHA protocol, where no two users transmit at the same time [5], [6]. CSMA has many applications, particularly in public areas, such as university campuses, train stations, and airports where many users try to connect to one access point. The same is true of private venues such as office buildings. In such cases CSMA can be applied to organize access to the main core point.

The ideal scenario for wireless communication networks employing the CSMA protocol is that no two sources send at the same time. However, in the complicated media applications in modern, urban, cities collisions usually happen. One of the most common reasons is hidden nodes. Two, or more, nodes are hidden, when they are not in range of each other, i.e. there is no access or direct connection between them [7]. However, in wireless networks, hidden nodes can occur where the sources are in range of each other, but cannot hear each other. For instance, when one or more source is hidden from the others due, for example, to restrictive environments such as the presence of building or any kind of vehicle, interference between users occurs, and leads to a reduction in system performance.

The effect of the hidden node problem on visible light communication has been studied in [8], where the authors simulate the uplink system performance using slotted CSMA random access procedure. The performance analysis of IEEE 802.11 ac distributed coordination function with hidden nodes has been proposed in [9]. The effect of hidden and exposed nodes on the performance of high throughput mesh networks was introduced in [10], where the authors designed improved channel access mechanisms, utilizing frame aggregation with high throughput wireless standards.

In this paper, we analyse the performance of a relay connecting a group of independent sources to their destination, employing a random CSMA type protocol. The sources are isolated from their destination, and the only way they can reach it is through the common relay, which acts as a blind $\mathrm{AF}$ relay. Sources are randomly distributed around the relay, and experience Rayleigh fading in their channels. We develop a new and more accurate model for multiple access interference at both the relay and destination, taking into account (in addition to the random sources locations) the effects of fading and 
thermal noise at both the relay and the destinations. Moreover, the effect of the hidden nodes on the system performance is considered as a randomly occurring factor. To the best of the authors' knowledge, there is little prior work which considers the effect of hidden nodes as random parameter.

We derive a new analytical expression for the overall spectral efficiency (SE) which can be used to estimate the throughput of the CSMA-based relay in Rayleigh fading channels, and to study the impact of the different system parameters on system efficiency. The signal-to-interference plus noise ratio (SINR) in this model is a ratio of a large number of random variables. However, the direct approach to finding the overall SE may require at least $(3 K+2)$-fold numerical integrations, which is generally difficult to obtain. We use Lemma1 (see below) to evaluate this average, where the result includes a simple mathematical equation that can be easily evaluated.

We derive explicit expressions for the SE of these models, where the new results are expressed in terms of the weights and abscissas of a Laguerre orthogonal polynomial. Furthermore, simulations are provided to validate our analysis. The results show that the SE of the proposed system is affected by factors such as number of sources, source status, thermal noise, and the radius of the coverage area.

The remainder of this paper is organized as follows. In Section II, the system model is briefly described. The problem formulation is explained in Section III. The SE analysis and the exact-form are presented in Section IV. In Section V, the results are presented and discussed. Finally, conclusions are drawn in Section VI.

Notations: Bold lower case letters denote vectors, E[.] denotes the expectation operator, $\operatorname{Pr}($.$) denotes the probability$ function, |.| denotes the absolute value, we use $\sim C N(0,1)$ to stand for a circularly symmetric complex Gaussian distribution with zero mean and variance 1.

\section{System ModeL}

The system model under consideration is shown in Fig. 1; there are $K$ sources each communicating with the destination through a common relay. The sources are distributed uniformly in the service area around the relay, and the service area is considered as a circle with radius $A$. There are no direct links between the sources and the destinations because the channel between them is not reliable due to strong shadowing effects, such as inside buildings and tunnels, or deep fading. The sources, destination, and the relay are each equipped with single antenna. Carrier sense multiple access (CSMA) protocol operates between the sources and relay, while the sources share the same frequency band. Each source has a random location, and the distance between each source and the relay $r$ is represented by a random variable with distribution $f(r)=2 r / A^{2},(r \leq A)$. When the source $i$ is randomly chosen to communicate with its destination, the probability of its transmitted frames being successfully transmitted is unity. The communication between the sources and destination is divided into two phases. In first phase, the source wishing

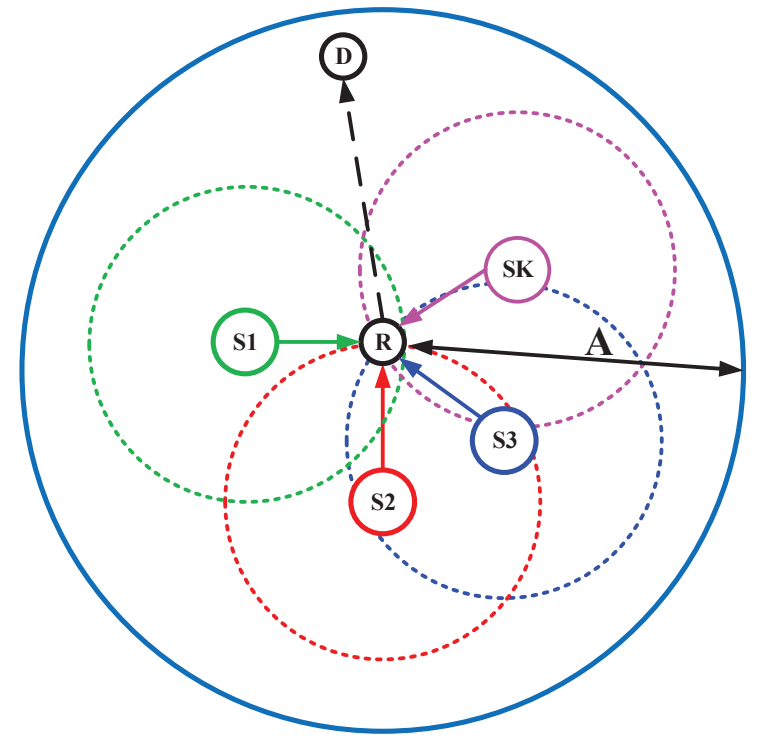

Fig. 1: System Model

to transmit its signal first listens to the channel to see if the another source is sending (carrier sense). If the channel is free, the source will send its signal, and if it is busy, will wait for a random time before trying again (Non-persistent). The received composite signal at the relay can be expressed as

$$
y_{r}=\sum_{k=1}^{K} \sqrt{p_{k}} h_{k} \delta_{k} r_{k}^{-\frac{\alpha}{2}} x_{k}+n_{g}
$$

where $p_{k}$ is the transmitted power by source $k, x_{k}$ is the transmitted symbol with unit power, $h_{k}$ is the complex channel gain between source $k$ and the relay, $r_{k}$ is the random distance between source $k$ and the relay, $\alpha$ is the path loss exponent, $n_{g}$ is the additive white Gaussian noise (AWGN) at the relay, with zero mean and $N_{g}$ variance, and $\delta_{k}$ represents the status of each source where

$$
\delta_{k}=\left\{\begin{array}{cc}
1 & \text { if source } k \text { ishidden } \\
0 & \text { otherwise }
\end{array}\right.
$$

The relay will receive $y_{r}$ and amplify it by gain $G$. In order to satisfy the peak power concentration, the gain of the relay is given as

$$
G=\sqrt{\frac{p_{g}}{\sum_{k=1}^{K} p_{k}\left|h_{k}\right|^{2} \delta_{k} r_{k}^{-\alpha}+N_{g}}}
$$

where $p_{g}$ is the transmitted power of the relay. In the second hop, the relay forwards the composite signal received from all sources. The received signal at the destination $i$ from $i^{\text {th }}$ 
source can be expressed as

$$
\begin{aligned}
& y_{i}=G g_{i} l_{i}^{-\frac{\alpha}{2}} \\
& \times\left(\left(\sqrt{p_{i}} h_{i} r_{i}^{-\frac{\alpha}{2}} x_{i}+n_{g}\right)+\left(\sum_{k \neq i}^{K} \sqrt{p_{k}} h_{k} \delta_{k} r_{k}^{-\frac{\alpha}{2}} x_{k}\right)\right)+n_{D}
\end{aligned}
$$

where $g_{i}$ is the complex channel gain between the relay and the destination $i, l_{i}$ is the distance between the relay and the destination $i$, and $n_{D}$ is the AWGN at the destination with zero mean and variance $N_{D}$. We assume that the destinations are randomly distributed around the relay. The SINR at destination $i\left(\mathrm{SINR}_{i}\right)$ can be written as

$$
\operatorname{SINR}_{i}=\frac{p_{i}\left|h_{i}\right|^{2}\left|g_{i}\right|^{2} r_{i}^{-\alpha} l_{i}^{-\alpha}}{\left|g_{i}\right|^{2} l_{i}^{-\alpha}\left(N_{g}+\sum_{k \neq i}^{K} p_{k}\left|h_{k}\right|^{2} \delta_{k} r_{k}^{-\alpha}\right)+N_{D} / G^{2}} .
$$

Substituting the gain of the relay as given in (3) into the expression for $\mathrm{SINR}_{i}$ in (5), we get (6), which shown at the top of next page.

Rewriting Equation (6), the final form of $\operatorname{SINR}_{i}$ can be expressed as

$$
\mathrm{SINR}_{i}=\frac{\frac{p_{i}\left|h_{i}\right|^{2} r_{i}^{-\alpha}}{\sum_{k \neq i}^{K} p_{k}\left|h_{k}\right|^{2} \delta_{k} r_{k}^{-\alpha}+N_{g}} \frac{p_{g}\left|g_{i}\right|^{2} l_{i}^{-\alpha}}{N_{D}}}{\frac{p_{g}\left|g_{i}\right|^{2} l_{i}^{-\alpha}}{N_{D}}+\frac{p_{i}\left|h_{i}\right|^{2} r_{i}^{-\alpha}}{\sum_{k \neq i}^{K} p_{k}\left|h_{k}\right|^{2} \delta_{k} r_{k}^{-\alpha}+N_{g}}+1} .
$$

\section{Problem Formulation}

In communication systems, the SE describes the data rate that can be achieved for a specific bandwidth. In this section, we derive the achievable SE of the two-phase relay system which can be expressed as

$$
\Re=\frac{1}{2} \mathrm{E}\left\{\sum_{k=1}^{K}\left[\log _{2}\left(1+\operatorname{SINR}_{k}\right)\right]\right\}
$$

where $\left[\log _{2}\left(1+\mathrm{SINR}_{k}\right)\right]$ is the instantaneous SE of the source $k$, and the factor $\frac{1}{2}$ comes from the fact that communication between the source and destination is performed in two phases [11]-[13]. As we assume that users are statically identical, the overall SE of this model can be written as

$$
\Re=\frac{K}{2} \mathrm{E}\left\{\left[\log _{2}\left(1+\mathrm{SINR}_{1}\right)\right]\right\}
$$

where $\mathrm{SINR}_{1}$ is the SINR of the first user; The expectation in (9) is with respect to the random variable $\mathrm{SINR}_{1}$, which is a ratio containing a large number of random variables. It is difficult to derive a closed-form expression for its distribution. The overall SE of our proposed model can be found from (9), where we need to find the expectation of this equation with respect to the following set of $(3 K+2)$ random variables: $\mathbf{h}=\left\{h_{1}, h_{2}, \ldots \ldots \ldots, h_{K}\right\}, \mathbf{r}=\left\{r_{1}^{-\alpha}, r_{2}^{-\alpha}, \ldots . ., r_{K}^{-\alpha}\right\}, \boldsymbol{\delta}=$ $\left\{\delta_{1}, \delta_{2}, \ldots \ldots \ldots, \delta_{K}\right\}, g_{i}$, and $l_{i}$. The direct approach to compute this expectation is difficult in general, as it may require the computation of $(3 K+2)$-fold convolution integrals. In this paper we use the simple and useful Lemma1 to evaluate the averaging in (9).
Lemma 1. : [14, Eq. (9)] In the special case where two random variables $U$ and $V$ are independent. it follows that

$$
\begin{aligned}
\mathrm{E}\left[\ln \left(1+\frac{U V}{1+U+V}\right)\right]= \\
\quad \int_{0}^{\infty} \frac{1}{z}\left(1-M_{U}(z)\right)\left(1-M_{V}(z)\right) e^{-z} d z
\end{aligned}
$$

where $M_{U}(z)=\mathrm{E}\left[e^{-z U}\right]$ and $M_{V}(z)=\mathrm{E}\left[e^{-z V}\right]$ are the moment generation functions of $U$ and $V$, respectively. From (10), the overall SE in (9) can be obtained as

$$
\begin{aligned}
\Re= & \frac{K}{2}\left(\log _{2} e\right) \\
& \times \mathrm{E}\left[\int_{0}^{\infty} \frac{1}{z}\left(1-M_{U}(z)\right)\left(1-M_{V}(z)\right) e^{-z} d z\right] .
\end{aligned}
$$

Equation (11) can be expressed in terms of the weights and abscissas of a Laguerre orthogonal polynomial

$$
\begin{aligned}
\Re= & \frac{K}{2}\left(\log _{2} e\right) \\
& \sum_{n=1}^{N} \xi_{n} \mathrm{E}\left\{\left(1-M_{U}\left(\beta_{n}\right)\right)\left(1-M_{V}\left(\beta_{n}\right)\right)\right\}+R_{N}
\end{aligned}
$$

where $\beta_{n}$ and $\xi_{n}$ are the sample points and the weights factors of the Laguerre polynomial, respectively, tabulated in [15, eq. (25.4.45)]. The remainder, $R_{N}$, is sufficiently small for $N \geq$ 15 , that (12) provides an efficient numerical evaluation of the required SE. In the next section, we evaluate (12) in case of Rayleigh fading, and we show how we can use Lemma 1 to simplify the above equation.

\section{SPECTRAL EFFICIENCY ANALYSIS}

In the first scenario, all channels are assumed to be subject to independent and identical distributed complex Gaussian fading, with zero mean and unit variance, e.g., $h_{i}, g_{i} \backsim C N(0,1)$, where $i=\{1,2, \ldots, K\}$. As a result, the magnitude of channels, $h_{i}$ and $g_{i}$, follows a Rayleigh distribution. Therefore, the distribution of power channel gains, $\left|h_{i}\right|^{2}$ and $\left|g_{i}\right|^{2}$ becomes exponential distribution. To find the SE, we need to find $M_{U}(z)$ and $M_{V}(z)$. Let $U=\frac{p_{i}\left|h_{i}\right|^{2} r_{i}^{-\alpha}}{\sum_{k \neq i}^{K} p_{k}\left|h_{k}\right|^{2} \delta_{k} r_{k}^{-\alpha}+N_{g}}$ and $V=\frac{p_{g}\left|g_{i}\right|^{2} l_{i}^{-\alpha}}{N_{D}}$. Without any loss of generality we assume equal transmission of power for all sources. Therefore, $\gamma_{\mathrm{g}}=\frac{p_{i}}{N_{0}}$ and $\gamma_{\mathrm{D}}=\frac{p_{g}}{N_{0}}$, where $\gamma_{\mathrm{g}}$ and $\gamma_{\mathrm{D}}$ are the signal to noise ratio (SNR) at the relay and destination, respectively.

\section{A. The $M G F$ of $U$}

As mentioned earlier, $U$ is a ratio containing a large number of random variables. Therefore, to find $M_{U}(z)$, we need to find the distribution of $U$. The complementary cumulative distribution function (CCDF) is

$$
\operatorname{Pr}(U>u)=\operatorname{Pr}\left(\frac{\left|h_{i}\right|^{2} r_{i}^{-\alpha}}{\sum_{k \neq i}^{K}\left|h_{k}\right|^{2} \delta_{k} r_{k}^{-\alpha}+a}>u\right)
$$




$$
\operatorname{SINR}_{i}=\frac{p_{i}\left|h_{i}\right|^{2} P_{g}\left|g_{i}\right|^{2} r_{i}^{-\alpha} l_{i}^{-\alpha}}{p_{g}\left|g_{i}\right|^{2} l_{i}^{-\alpha}\left(N_{g}+\sum_{k \neq i}^{K} p_{k}\left|h_{k}\right|^{2} \delta_{k} r_{k}^{-\alpha}\right)+N_{D}\left(\sum_{k=1}^{K} p_{k}\left|h_{k}\right|^{2} \delta_{k} r_{k}^{-\alpha}+N_{g}\right)} .
$$

where $a=\frac{1}{\gamma}$. Therefore (13) can be rewritten as

$$
\operatorname{Pr}(U>u)=\operatorname{Pr}\left(\left|h_{i}\right|^{2}>u r_{i}^{\alpha}\left(\sum_{k \neq i}^{K}\left|h_{k}\right|^{2} \delta_{k} r_{k}^{-\alpha}+a\right)\right) .
$$

Equation (14) contains more than one random variable, therefore we condition firstly on $(\mathbf{h}, \mathbf{r}, \boldsymbol{\delta})$ to get the conditional $\mathrm{CCDF}$

$$
\begin{aligned}
& \operatorname{Pr}(U>u \mid \mathbf{h}, \mathbf{r}, \boldsymbol{\delta})= \\
& \quad \operatorname{Pr}\left(\left|h_{i}\right|^{2}>u r_{i}^{\alpha}\left(\sum_{k \neq i}^{K}\left|h_{k}\right|^{2} \delta_{k} r_{k}^{-\alpha}+a\right) \mid \mathbf{h}, \mathbf{r}, \boldsymbol{\delta}\right) .
\end{aligned}
$$

Equation (15) is conditional on $(\mathbf{h}, \mathbf{r}, \boldsymbol{\delta})$. Since $\left|h_{i}\right|^{2}$ has an exponential distribution

$$
\operatorname{Pr}(U>u \mid \mathbf{h}, \mathbf{r}, \boldsymbol{\delta})=e^{-u r_{i}^{\alpha}\left(\sum_{k \neq i}^{K}\left|h_{k}\right|^{2} \delta_{k} r_{k}^{-\alpha}+a\right) .}
$$

Since the sources are independent, the random variables $\left|h_{k}\right|^{2}, r_{k}, \delta_{k}$ for $k=1,2, \ldots, K$ are mutually independent random variables. Average the independent random variables $\left\{\left|h_{k}\right|^{2}, r_{k}, \delta_{k}, k=1,2, \ldots, K, k \neq i\right\}$, we obtain from (16)

$$
\begin{gathered}
\operatorname{Pr}(U>u)=\mathrm{E}\left[e^{-u r_{i}^{\alpha}\left(\sum_{k \neq i}^{K}\left|h_{k}\right|^{2} \delta_{k} r_{k}^{-\alpha}+a\right)}\right]= \\
\prod_{k \neq i}^{K} \mathrm{E}\left[e^{-u r_{i}^{\alpha} \sum_{k \neq i}^{K}\left|h_{k}\right|^{2} \delta_{k} r_{k}^{-\alpha}}\right] e^{-u a r_{i}^{\alpha}}= \\
\prod_{k \neq i}^{K} \mathrm{E}\left[\frac{1}{1+u r_{i}^{\alpha} \delta_{k} r_{k}^{-\alpha}}\right] e^{-u a r_{i}^{\alpha}}
\end{gathered}
$$

The term $\delta_{k}$ is a random variable representing the status of each source, whether it is hidden or not. In this system, we model $\delta_{k}$ by independent Bernoulli random variable, where the probability, $\operatorname{Pr}\left(\delta_{i}=1\right)=\rho$ and $\operatorname{Pr}\left(\delta_{i}=0\right)=1-\rho$. Therefore, the probability $\operatorname{Pr}(U>u)$, in (17) can be shown to be

$$
\operatorname{Pr}(U>u)=\prod_{k \neq i}^{K} \mathrm{E}\left[1+\rho-\frac{\rho}{1+u r_{i}^{\alpha} r_{k}^{-\alpha}}\right] e^{-u a r_{i}^{\alpha}}
$$

where $\rho$ is the probability that the source is hidden or not, and takes a value in the range $0 \leq \rho \leq 1$. The expectation in (18) is with respect to the distance $r_{k}$. As far as the distribution of $r_{k}$ is concerned, it can be shown that their probability density function (PDF) is $f(r)=2 r / A^{2}, r \leq A$. Equation (17) can now be written as

$$
\begin{aligned}
& \operatorname{Pr}\left(U>u \mid r_{i}\right)= \\
& \quad\left(\int_{0}^{A} 1-\rho+\frac{\rho}{1+u r_{i}^{\alpha} r_{k}^{-\alpha}} \frac{2 r_{k}}{A^{2}} d r_{k}\right)^{K-1} e^{-u a r_{i}^{\alpha}}
\end{aligned}
$$

The integral in (19) can be evaluated in closed form to obtain

$$
\begin{aligned}
& \operatorname{Pr}\left(U>u \mid r_{i}\right)= \\
& \left(1-\rho+\rho\left(1-{ }_{2} F_{1}\left(1 ; \frac{2}{\alpha} ; \frac{2+\alpha}{\alpha} ;-\frac{A^{\alpha}}{u r_{i}^{\alpha}}\right)\right)\right)^{K-1} \\
& \times e^{-u a r_{i}^{\alpha}}
\end{aligned}
$$

where ${ }_{2} F_{1}(a ; b ; u)$ is the confluent hypergeometric function. On averaging $r_{i}$ we obtain

$$
\begin{aligned}
& \operatorname{Pr}(U>u \mid K)= \\
& \qquad \begin{aligned}
\int_{0}^{A}\left(1-\rho_{2} F_{1}\left(1 ; \frac{2}{\alpha} ; \frac{2+\alpha}{\alpha}\right.\right. & \left.\left.;-\frac{A^{\alpha}}{u r_{i}^{\alpha}}\right)\right)^{K-1} \\
& \times \frac{2 r_{i}}{A^{2}} e^{-u a r_{i}{ }^{\alpha}} d r_{i} .
\end{aligned}
\end{aligned}
$$

The cumulative distribution function (CDF) of $U$ is given by $1-\operatorname{Pr}(U>u)$. Integration $M_{U}(z)=\mathrm{E}\left[e^{-z U}\right]$, by parts we obtain

$$
\begin{aligned}
& M_{U}(\mathrm{z})=\int_{0}^{\infty} e^{-z U} f(U) d U \\
& =1-z \int_{0}^{\infty} \int_{0}^{A}\left(1-\rho_{2} F_{1}\left(1 ; \frac{2}{\alpha} ; \frac{2+\alpha}{\alpha} ;-\frac{A^{\alpha}}{u r_{i}^{\alpha}}\right)\right)^{K-1} \\
& e^{-u\left(z+\frac{r_{i}^{\alpha}}{\gamma}\right)} \frac{2 r_{i}}{A^{2}} d u d r_{i} .
\end{aligned}
$$

Equation (22) can also be expressed in terms of the weights and abscissas of a Laguerre orthogonal polynomial

$$
\begin{aligned}
M_{U}(z)= & \\
1-z \sum_{b=1}^{B} \mu_{b} \int_{0}^{A} \gamma r_{i}^{-\alpha}(1- & \left.\rho_{2} F_{1}\left(1 ; \frac{2}{\alpha} ; \frac{2+\alpha}{\alpha} ;-\frac{A^{\alpha}}{\lambda_{b} \gamma}\right)\right)^{K-1} \\
& \times e^{-z \lambda_{b} \gamma r_{i}^{-\alpha}} \frac{2 r_{i}}{A^{2}} d r_{i}+R_{B}
\end{aligned}
$$

where $\lambda_{b}$ and $\mu_{b}$ are the sample points and the weights factors of the Laguerre polynomial, respectively, tabulated in [15, eq. (25.4.45)]. For $B \geq 15$, the remainder, $R_{B}$, is sufficiently small to be ignored.

\section{B. The $M G F$ of $V$}

For $M_{V}(z)$, where $V=\frac{P_{g}\left|g_{i}\right|^{2} l_{i}^{-\alpha}}{N_{D}}=\gamma_{\mathrm{D}}\left|g_{i}\right|^{2} l_{i}^{-\alpha}, M_{V}(z)$ can be obtained as

$$
\begin{aligned}
M_{V}\left(z \mid l_{i}^{-\alpha}\right)= & \mathrm{E}\left[e^{-z V}\right]= \\
& \int_{0}^{\infty} e^{-z V} \lambda e^{-\lambda V} d V=\frac{1}{1+z_{\gamma_{\mathrm{D}}} l_{i}^{-\alpha}} .
\end{aligned}
$$

The term $l_{i}$ represents the random distance between the gateway and the destination $i$, which has a distribution given by $f(l)=\frac{2 l}{A^{2}}, l \leq A$. From (17) it follows that 


$$
\begin{aligned}
& M_{V}(z)= \\
& \int_{0}^{A} \frac{1}{1+z_{\gamma_{\mathrm{D}}} l_{i}^{-\alpha}} \frac{2 l_{i}}{A^{2}} d l_{i}=1-{ }_{2} F_{1}\left(1 ; \frac{2}{\alpha} ; \frac{2+\alpha}{\alpha} ;-\frac{A^{\alpha}}{z \gamma_{\mathrm{D}}}\right) .
\end{aligned}
$$

Substituting (25) and (23) into (12) leads to the tractable explicit expression for the overall average SE in Rayleigh fading. The new result includes only one integration. It is worth remembering that according to (9), the direct method to find the overall $\mathrm{SE}$ requires a $(3 K+2)$-fold convolution integral.

\section{Numerical And Simulation Results}

In this section, the SE achieved by the multiple-access AF relay is evaluated using Monte-Carlo simulations, and compared to the derived asymptotic results In addition, the complex channels gains are modelled as Rayleigh distributions. It can be assumed that $\gamma_{D}=\gamma$, without loss of generality. Different graphical plots of SE are presented below, corresponding to various numbers of users, $\gamma$ values, radius of the coverage area, and source status.

Table I: SIMULATION PARAMETERS
\begin{tabular}{ll}
\hline Parameter & Value \\
\hline Path-loss exponent $\alpha$ & 3 \\
Radius area $A$ & $1 \mathrm{~km}$ \\
SNR $(\gamma)$ & $10 \mathrm{~dB}$ \\
Number of sources & 10 \\
\hline
\end{tabular}

In Fig. 2, the SE is plotted in bits/s/Hz, as function of the number of sources for different probability that the source is hidden $(\rho)$. From this figure, it is clear that, for all values of $\rho$, as the number of sources increase, the SE increases correspondingly until it attains a maximum value, after which there is a gradual decrease in the value of the SE. This is because increasing the number of sources, increases interference effects which leads to drop in the system performance. It can be also be seen that decreasing the value of $\rho$ gives better performance (greater value of SE) due to the sources of useful signals increasing, which leads to a better SINR value. However, decreasing the value of $\rho$ shifts the peak value of the SE to a higher value of $K$.

Fig. 3, shows the SE as a function of source status (probability that source is hidden) with increasing signal to noise ratio $(\gamma$ $=10,20$, and $30 \mathrm{~dB}$ ). It can be seen that for low values of $\rho$, improving signal to noise ratio, so that the effect of the noise is limited, improves system performance and gives a substantial increase in SE. As $\rho$ increases, the probability of hidden nodes is increased, interference is increased and the SE decreases asymptotically. As would be expected, the effect of $\gamma$ on performance of the system is positive, with higher $\gamma$ leading to better performance.

Fig. 4, shows the SE as a function of SNR. It is clear that for all values of $\gamma$ the SE decreases with increase in the probability that the source is hidden $(\rho)$. For example, at high

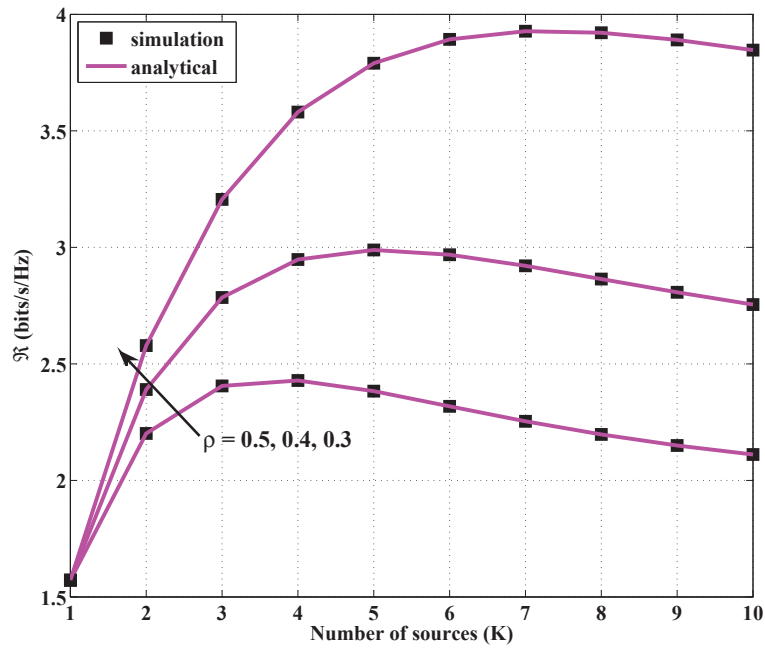

Figure 2: SE as function in number of sources $(K)$

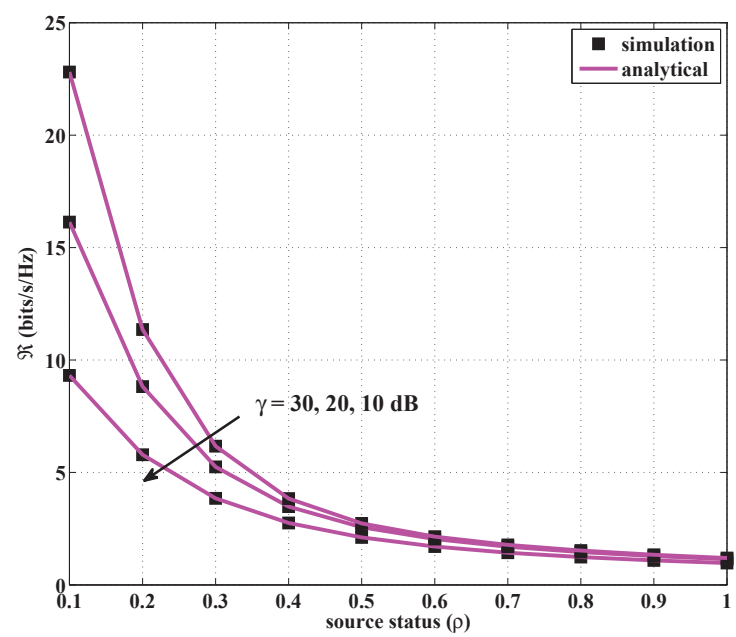

Figure 3: SE as function in source status $(\rho)$

values of SNR $(\gamma=30 \mathrm{~dB})$, we see that decreasing $\rho$ from 1.0 to 0.5 increases the system performance dramatically by about $1.5 \mathrm{bits} / \mathrm{s} / \mathrm{Hz}$. This is interpreted as showing that, in interference limited systems the noise contribution becomes minimal $(1 / \gamma \rightarrow 0)$, and that as $\rho$ decreases the interference in the system decreases.

In Fig. 5, the SE is plotted as function of the radius $(A)$ of a circular coverage area. The smaller the area covered the greater the value of the SE. As $A$ increases, the path loss distance is also increased, which leads to a decrease in the power of the useful signal. It can be seen that the higher the value of $\rho$ the worse the system's performance and that SE decreases asymptotically to zero with increase in $A$.

\section{CONCLusions.}

In this paper, we have analyzed in detail the performance of a relay connecting a group of independent sources to their 


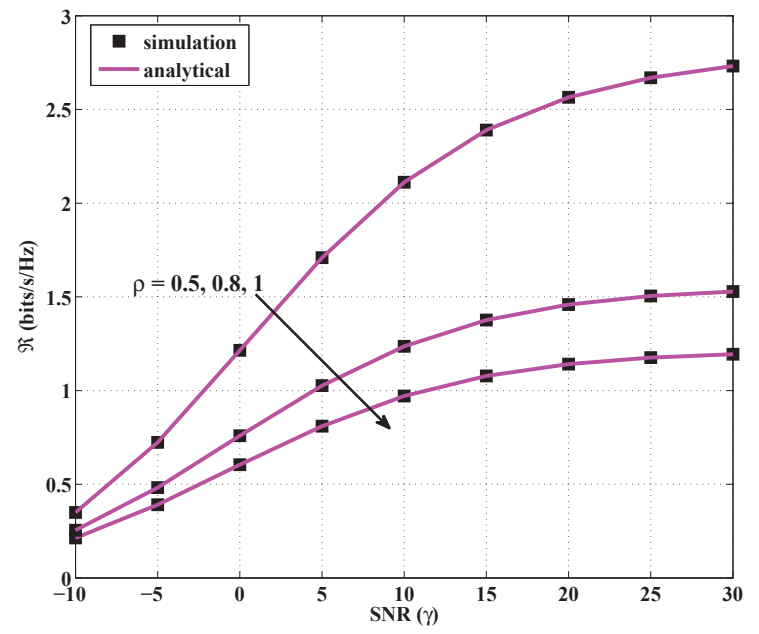

Figure 4: SE as function in SNR $(\gamma)$

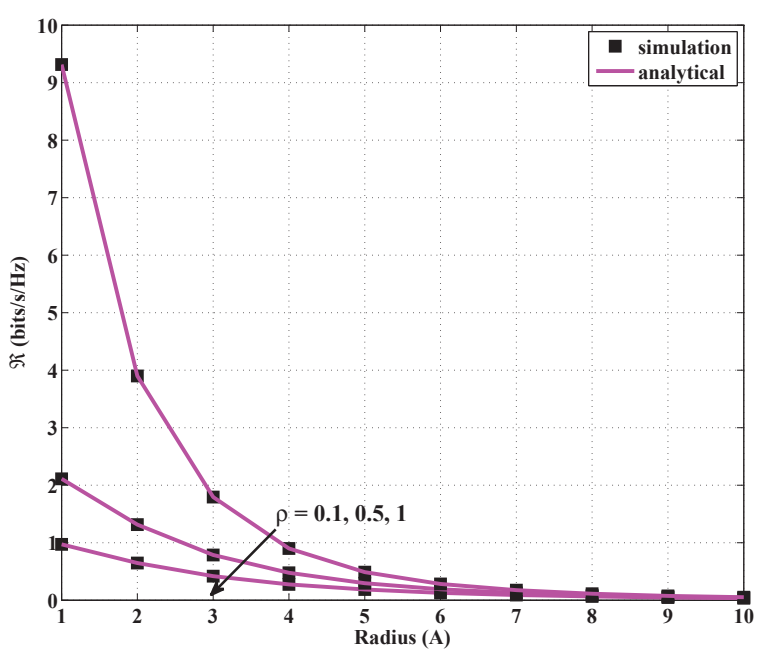

Figure 5: SE as function in radius $(A)$

destination, employing a random CSMA protocol. Moreover, the effect of hidden nodes on system performance (SE) is considered. The sources are randomly distributed around the relay within a circular area of radius $A$, and the destination are equipped with single antenna. We have derived analytical expressions for the overall SE, where we assumed the channels between the sources, gateway, and destination are subject to Rayleigh fading channels. The performance analysis of this model is difficult to obtain in general using the direct approach, as it may require the computation of $(3 K+2)$-fold convolution integral to find the distribution of the $\mathrm{SINR}_{1}$. As result, we used a useful Lemma 1 to evaluate the performance of the model and produce a relatively simple equation that can be more easily evaluated. Our result reflects the fact that the mathematical analysis and the useful Lemma 1 can be used in different system models. We validated the accuracy of our results with Monte Carlo simulations. We also examined the impact of increasing the number of sources on the performance of such a system and showed that, as the number of sources increased, the SE also increased, and then saturated. In addition, we used the model to evaluate the effect of important factors such as SNR, and source status on the system performance.

\section{REFERENCES}

[1] P. S. Lu, X. Zhou, and T. Matsumoto, "Outage probabilities of orthogonal multiple-access relaying techniques with imperfect source-relay links," IEEE Trans. Wireless Commun., vol. 14, no. 4, pp. 2269-2280, 2015.

[2] L. Liu, Y. Li, Y. Su, and Y. Sun, "Quantize-and-forward strategy for interleave-division multiple-access relay channel," IEEE Trans. Veh. Technol., vol. 65, no. 3, pp. 1808-1814, 2016.

[3] A. Molisch, Multiple Access and the Cellular Principle. Wiley-IEEE Press, 2011, pp. 365-385. [Online]. Available: http://ieeexplore.ieee.org/xpl/articleDetails.jsp?arnumber $=5635469$

[4] A. Indira Balan and V. Tiruchirai Gopalakrishnan, "Analysis of carrier sense multiple access protocols for channels supporting multi-packet reception," IEEE IET Commun., vol. 9, no. 4, pp. 468-475, 2015.

[5] H.-H. Choi, J.-M. Moon, I.-H. Lee, and H. Lee, "Carrier sense multiple access with collision resolution," IEEE Commun. Lett., vol. 17, no. 6 , pp. 1284-1287, 2013.

[6] S. Pollin, M. Ergen, S. Ergen, B. Bougard, L. Der Perre, I. Moerman, A. Bahai, P. Varaiya, and F. Catthoor, "Performance analysis of slotted carrier sense ieee 802.15.4 medium access layer," IEEE Trans. Wireless Commun, vol. 7, no. 9, pp. 3359-3371, 2008.

[7] S. N. P. V. V. Kapadia and R. H. Jhaveri, "Comparative study of hidden node problem and solution using different techniques and protocols," $J$. of Computing, vol. 2, no. 3, pp. 65-67, Mar. 2010.

[8] C. Ley-Bosch, I. Alonso-Gonzalez, D. Sanchez-Rodriguez, and M. A. Quintana-Suarez, "Analysis of the effects of the hidden node problem in ieee 802.15.7 uplink performance," in 2015 Int. Conf. Computer Inf. Telecomm. Syst. (CITS), 2015, pp. 1-5.

[9] Z. Chang, O. Alanen, T. Huovinen, T. Nihtila, E. H. Ong, J. Kneckt, and T. Ristaniemi, "Performance analysis of ieee 802.11ac dcf with hidden nodes," in IEEE Veh. Technol. Conf. (VTC), 2012, pp. 1-5.

[10] S. Chakraborty, S. Nandi, and S. Chattopadhyay, "Alleviating hidden and exposed nodes in high-throughput wireless mesh networks," IEEE Trans. Wireless Commun., vol. 15, no. 2, pp. 928-937, 2016.

[11] B. Zhong, X. Zhang, Y. Li, Z. Zhang, and K. Long, "Impact of partial relay selection on the capacity of communications systems with outdated CSI and adaptive transmission techniques," in IEEE Wireless Commun. Netw. Conf. (WCNC), Apr. 2013, pp. 3720-3725.

[12] T. Liu and C. Yang, "Spectral efficiency comparison between MCCDMA two-hop relay systems with different channel information," IEEE Trans. Veh. Technol., vol. 61, no. 8, pp. 3603-3614, 2012.

[13] A. Mahmud and K. A. Hamdi, "Unified framework for spectral efficiency analysis of AF relay system," in IEEE Wireless Commun. Netw. Conf. (WCNC), 2015, pp. 1695-1700.

[14] A. Alfitouri and K. A. Hamdi, "Multiple-access capabilities of amplifyand-forward relaying," in IEEE Global Commun. Conf. (GLOBECOM), 2015, pp. 1-6.

[15] M. Abramowitz and I. A. Stegun, Handbook of Mathematical Functions. New York, NY, USA: Dover, 1965. 